\title{
A THEORETICAL ANALYSIS OF EQUIBIAXIAL DEFORMATION TEXTURE UNDER NON-PRESCRIBED STRAIN PATH
}

\author{
W. B. LEE and K. C. CHAN \\ Department of Manufacturing Engineering, Hong Kong Polytechnic, Hung Hom, \\ Hong Kong
}

(Received 17 April 1990)

\begin{abstract}
A model is presented to calculate the rotation path of crystals under equibiaxial tension when the strain ratio is fixed (prescribed strain path) or when the strain ratio is allowed to adjust itself according to the external constraint and the current state of the plastic anisotropy of the material (nonprescribed strain path). It is found that the stability of grain orientation is related to the curvature of the strain path. There is a difference in the predicted equibiaxial deformation texture for the two types of strain paths. Grain orientations which are unstable under a linear strain path may become stable under a non-linear or varying strain path.
\end{abstract}

KEY WORDS Biaxial deformation, Model calculation, Strain path, Orientation path, Texture evolution, FCC sheet metals.

\section{INTRODUCTION}

When a polycrystalline metal is plastically deformed, a change of grain orientation occurs. For high stacking fault energy metals and alloys deformed at room temperature, the main deformation mechanism is slip by dislocations. The crystal rotation depends on the orientation of the slip systems relative to the imposed shape change and the strain path. As there is only a limited number of slip systems available, the grains may rotate towards a limited number of stable end orientations, thus creating a crystallographic texture. The main loading paths considered in early texture theories (Sachs, 1928, Taylor, 1938) are tension and plane strain compression. The Taylor model was extended by Bishop and Hill (1951a, 1951b) to include arbitrary imposed strains. Further refinements of the model were made by Bunge (1971) and Van Houtte and Aernoudt (1975). The effect of strain path on the slip rotation however has been relatively less studied.

The strain components that a material can take are related to the boundary conditions and the plastic anisotropy of the material. The boundary conditions will define the number of prescribed strain components for a given shape change. For example, under plane-strain conditions, the strain in the transverse direction is forbidden and a reaction stress will build up. The plastic anisotropy on the other hand will determine the value of the non-prescribed strain components. When a thin metal sheet is deformed under equibiaxial tension, the shear 
components are taken to be zero and the strain tensor $\epsilon$ is given by

$$
\boldsymbol{\epsilon}=d \boldsymbol{d \epsilon}\left[\begin{array}{ccr}
X & 0 & 0 \\
0 & 1-X & 0 \\
0 & 0 & -1
\end{array}\right]
$$

where $X$ is dimensionless parameter.

When an isotropic sheet is deformed under equibiaxial tension $\left(\sigma_{11}=\sigma_{22}\right)$, the ratio of the two principal strain components $\left(\epsilon_{22} / \epsilon_{11}\right)$ equals 1 . Even though the imposed stress ratio is 1 , the strain ratio of an anisotropic sheet metal will not equal 1. In addition, after a small strain increment $d \epsilon$, the grains may rotate to new orientations and the principal strain ratio will vary during deformation. In this paper, strain is regarded as a function of stress but the magnitude of this strain ratio cannot be predicted from the stress ratio based on phenomenological yield equations alone. If the principal strain components increase in proportion with external loading, the path is said to be linear or proportional. When the successive strain states vary, the path would be non-linear. The practice of assigning a fixed strain state for all subsequent deformation will give a strain path that is completely prescribed beforehand. If the strain path is fixed, the imposed stress has to be varied continuously. Strictly speaking, such a fixed strain ratio would not be valid even for an isotropic material as a texture will develop eventually during the course of plastic deformation. The strain components would continuously vary and adjust themselves according both to the imposed stress state and the current anisotropy of the material. The strain path determined in this manner will be called a non-prescribed one. In this paper, the equibiaxial texture obtained under the prescribed and non-prescribed type of strain path for fcc sheet metals are analysed.

\section{THE STRAIN PATH AND SLIP ROTATION}

A model for the theoretical prediction of the strain path of a polycrystalline sheet metal deformed under equibiaxial tension has been proposed by the authors (Chan and Lee, 1990) and some essential parts are outlined here to keep a self-consistency of this paper. The strain path is defined by the locus of the successive states of the cumulative strain followed by a material element. Elastic strain is neglected and the principal stress axes are assumed to be coincident with principal strain axes. A small cubic element in a metal sheet with its edges parallel to the rolling, transverse and normal directions will be deformed into a rectangular parallelopid. In biaxial stretching, the imposed stress is given by

$$
\boldsymbol{\sigma}=\left[\begin{array}{ccc}
\sigma_{11} & 0 & 0 \\
0 & \sigma_{22} & 0 \\
0 & 0 & 0
\end{array}\right]
$$

where $\sigma_{11}$ and $\sigma_{22}$ coincide with the rolling and transverse directions of the sheet respectively. The imposed stress ratio $\left(\sigma_{22} / \sigma_{11}\right)$ is assumed to be constant and the stress path is linear during the incremental computations. In equibiaxial tension, $\sigma_{22} / \sigma_{11}$ equals 1 . The corresponding strain tensor is given by Eq. (1) above. 
In equibiaxial stretching, $\sigma_{11}=\sigma_{22}$ but the magnitudes of $\epsilon_{11}$ and $\epsilon_{22}$ are unknown quantities that have to be determined from the anisotropic properties of the polycrystalline material. The sheet is supposed to consist of $N$ groups of grains each with volume fraction $f_{g}(g=1$ to $N)$ and orientation $\{h k l\}\langle u v w\rangle$. Each grain is assumed to undergo the same strain state as the polycrystal, i.e.,

$$
\epsilon_{i j}=\epsilon_{i j}^{g}
$$

To maintain equilibrium, the summation of the stresses of the individual grains must equal the imposed stress such that

$$
\sigma_{i j}=\sum_{g=1}^{N} f_{g} \sigma_{i j}^{g}
$$

$\sigma_{i j}^{g}$ is solved for each group of grains by applying the flow rule to an anisotropic yield function $\left(F\left(\sigma_{i j}\right)=0\right)$ referred to the specimen axes. The anisotropic continuum yield loci of the Continuum Mechanics of Textured Polycrystals (CMTP) (Montheillet et al., 1985) which is obtained by fitting a continuous curve to the crystallographic Bishop and Hill yield surface (Bishop and Hill 1951a, $1951 b$ ) is adopted here. Isotropic hardening of slip systems is assumed in the derivation of the yield surface. Since the crystallographic yield locus refers to the $\langle 100\rangle$ crystal axes of the grain, $\epsilon_{i j}$ has to be transformed into the cube axis by the relation

$$
\boldsymbol{\epsilon}_{c}^{g}=\mathbf{P} \in \overline{\mathbf{P}}
$$

where $\mathbf{P}$ is the transformation matrix.

The anisotropic yield locus of the CMTP is expressed as

$$
\begin{aligned}
F\left(S_{i j}\right)= & \alpha\left[\left|S_{11}-S_{22}\right|^{n}+\left|S_{22}-S_{33}\right|^{n}+\left|S_{33}-S_{11}\right|^{n}\right] \\
& +2 \beta\left[\left|S_{23}\right|^{n}+\left|S_{12}\right|^{n}+\left|S_{13}\right|^{n}\right]-1=0
\end{aligned}
$$

where $\alpha, \beta$ and $n$ are parameters that depend on the Gaussian distribution of the scatter width of the orientation (Bunge, 1969). The stress tensor is related to the strain tensor by applying the normality rule to the analytical yield function of Eq. (6) as follows

$$
\epsilon_{(c) i j}^{g}=\lambda \frac{\partial F\left(S_{(c) i j}^{g}\right)}{\partial S_{(c) i j}^{g}}
$$

By reducing the stress and strain tensors to the ones which contain only 5 independent components in the form of

$$
\begin{gathered}
\epsilon_{(c)}^{g}=\left[\left(\epsilon_{(c) 22}^{g}-\epsilon_{(c) 11}^{g}\right) / \sqrt{2}, \quad \epsilon_{(c) 33}^{g} \sqrt{(3 / 2)}, \epsilon_{(c) 23}^{g} \sqrt{2},\right. \\
\left.\epsilon_{(c) 31}^{g} \sqrt{2}, \epsilon_{(c) 12}^{g} \sqrt{2}\right],
\end{gathered}
$$

the yield function then becomes

$$
\begin{aligned}
F(\mathbf{S})= & \alpha 2^{-n / 2}\left[\left|2 S_{1}\right|^{n}+\left|S_{1}-3^{1 / 2} S_{2}\right|^{n}+\left|S_{1}+3^{1 / 2} S_{2}\right|^{n}\right] \\
& +\beta 2^{1-n / 2}\left[\left|S_{3}\right|^{n}+\left|S_{4}\right|^{n}+\left|S_{5}\right|^{n}\right]=1
\end{aligned}
$$

and the deviator stress tensor obtained from Eq. (7) is transformed back into the specimen axes as

$$
\mathbf{S}^{g}=\overline{\mathbf{P}} \mathbf{S}_{c}^{g} \mathbf{P}
$$


$X$ in Eq. (1) is varied until the deviatoric stress components $S_{i j}$ equal the imposed stress tensor for an incremental deformation of $d \epsilon$. $\epsilon$ then defines the strain path of the sheet element. The crystal rotation path depends on the orientation of the initial crystallographic orientation $\{h k l\}\langle u v w\rangle$ with respect to the specimen axes, the critical resolved shear stress of the slip systems and the strain tensor $\epsilon$ calculated from the above iteration procedures. A grain with initial orientation $\mathbf{T}$ will rotate to a new orientation $\mathbf{T}^{\prime}$ given by

and

$$
\mathbf{T}^{\prime}=\left(\mathbf{I}-\boldsymbol{\Omega}_{c}\right) \mathbf{T}
$$

$$
d \omega_{(c) i j}=(1 / 2) \sum_{s=1}^{12}\left(a_{j}^{s} b_{i}^{s}-a_{i}^{s} b_{j}^{s}\right) \gamma^{s}
$$

The shear strain $\gamma^{s}$ is related to the strain increment of $\epsilon_{(c) i j}^{g}$ by a matrix that denotes the direction cosines $\left(a_{i} b_{j}\right)$ of the slip systems with respect to the cube axis of the grain. There are many ways of choosing the 5 independent slip systems out of the 12 possible in fcc metals. The criterion for selecting the slip systems is based on Bishop and Hill's principle of maximum work (Bishop and Hill, 1951a, 1951b) for fully constrained deformation. Equal hardening of slip systems is assumed. The ambiguity in identifying the set of 5 slip system is resolved by solving the set which minimizes the second order plastic work as proposed by Renouard and Winterberger (RW) (1981). According to RW, an infinitesimal strain tensor is applied to each possible set of slip systems which will lead to a small crystal rotation. The new (second order) plastic work is then calculated. The set of slip systems corresponding to the minimum second order plastic work is selected. This approach has been successfully applied to the large strain deformation of aluminium crystals in plane strain compression (Driver et al., 1983). Bacroix et al. (1986) have compared this approach to the averaging (Van Houtte and Aernoudt, 1975) rate sensitivity (Kocks, 1975) and relaxed constraint (Honneff and Mecking, 1978) approaches. It is found that RW leads to better agreement with experimental observations than the averaging and rate sensitivity models.

The next strain state is calculated based on the new orientation $\mathbf{T}^{\prime}$ determined in Eq. (2). Repetition of the above procedures will yield a rotation path for each of the strain paths followed. The main difference in the calculation of the rotation path under a non-prescribed strain path and a prescribed strain path is that in the former case $\epsilon_{11}$ and $\epsilon_{22}$ are varied for each incremental step of deformation $(d \epsilon)$ but in the latter case they are assigned to be fixed.

\section{RESULTS AND DISCUSSIONS}

The rotation path for the texture components of an annealed commercially pure aluminium sheet deformed under a prescribed and non-prescribed strain path under balanced biaxial tension are simulated. The percentage of the different texture components of the aluminium sheet are taken from the orientation distribution functions (ODFs) in the published literature (Hansen and Jensen, 1986) and the texture is represented by $\{110\}\langle 112\rangle(35.09 \%)+\{123\}\langle 634\rangle(24.56 \%)+$ 


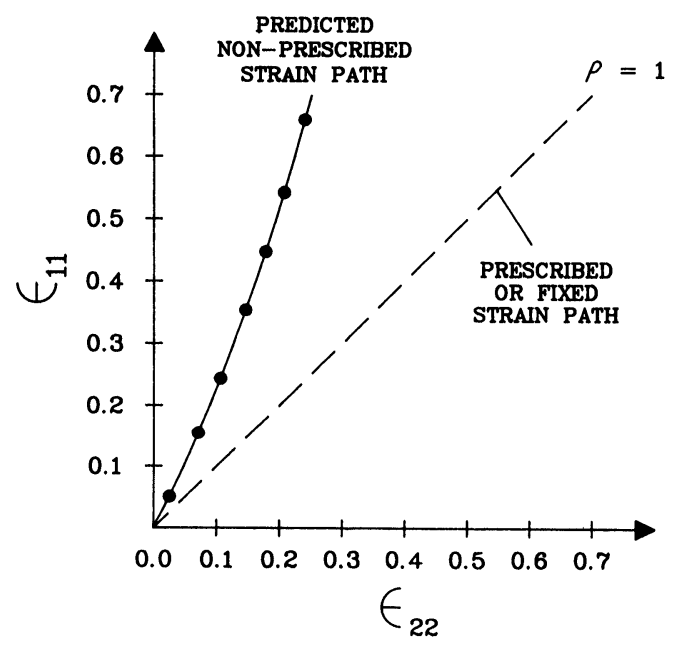

Figure 1 Predicted non-prescribed strain path of the C.P. aluminium sheet under equibiaxial tension.

$\{112\}\langle 111\rangle(5.26 \%)+\{100\}\langle 001\rangle(19.30 \%)+\{110\}\langle 001\rangle(15.79 \%)$. For each texture component, orientation spreads of $\pm 5^{\circ}$ and $\pm 10^{\circ}$ from the single orientations are considered. When the symmetrical orientations are taken into account, the total number of orientations in the simulated starting texture amounts to 260 . Random orientations in the aluminium sheet were not taken into account. For a scatter width of zero, the $n$ in the CMTP yield criterion (Eq. (6)) is 1.6 and $\alpha$ and $\beta$ equal to 0.46 and 0.51 respectively (Lequeu et al., 1986).

In the simulation, the parameter $X$ was varied between 0 and 1 in steps of 0.01 and $d \epsilon$ was taken to be 0.025 . An effective strain up to 0.8 was applied. Simulated strain paths for the C.P. aluminium sheet are shown in Figure 1. The strain path of the aluminium sheet is found to deviate a lot from the isotropic case (i.e., $\rho\left(\epsilon_{22} / \epsilon_{11}\right)=1$ ). A smaller differences of $15 \%$ in the two principal surface strains of an aluminium sheet in hydraulic bulging was reported by Ragab and Albas (1986). The discrepancy between the theoretical calculation made here and the experimental observations of Ragab and Albas is due to the small number of input orientations (i.e. only 260 orientations are considered in the calculation) and the scatter width is taken to be zero. With a more realistic input of the sheet orientations which take into account the random orientations, the predicted result will be expected to move closer to the experimental one. Another source of the discrepancy can be due to the fact that a hydraulic bulging test may not impose equibiaxial stresses (Vial and Hosford, 1983).

The rotation paths for the main texture components in the C.P. aluminium sheet are shown in Figure 2. There is a difference in the rotation path between the prescribed and non-prescribed strain paths. The simulated initial sheet texture is shown in Figure 3a. An effective strain of $\epsilon=0.8$ was applied to the sheet and the corresponding simulated $\{111\}$ pole figures of the equibiaxial deformation texture are shown in Figure $3 b \&$ c. The components of the two final textures (Figure $3 b \& \mathrm{c}$ ) consist of $\langle 110\rangle$ parallel to ND with minor peaks at orientations $\{110\}\langle 100\rangle$ and $\{110\}\langle 112\rangle$. This finding is in agreement with the experimental 


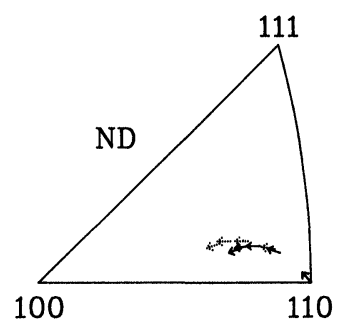

(a) $\{110\}<112>$
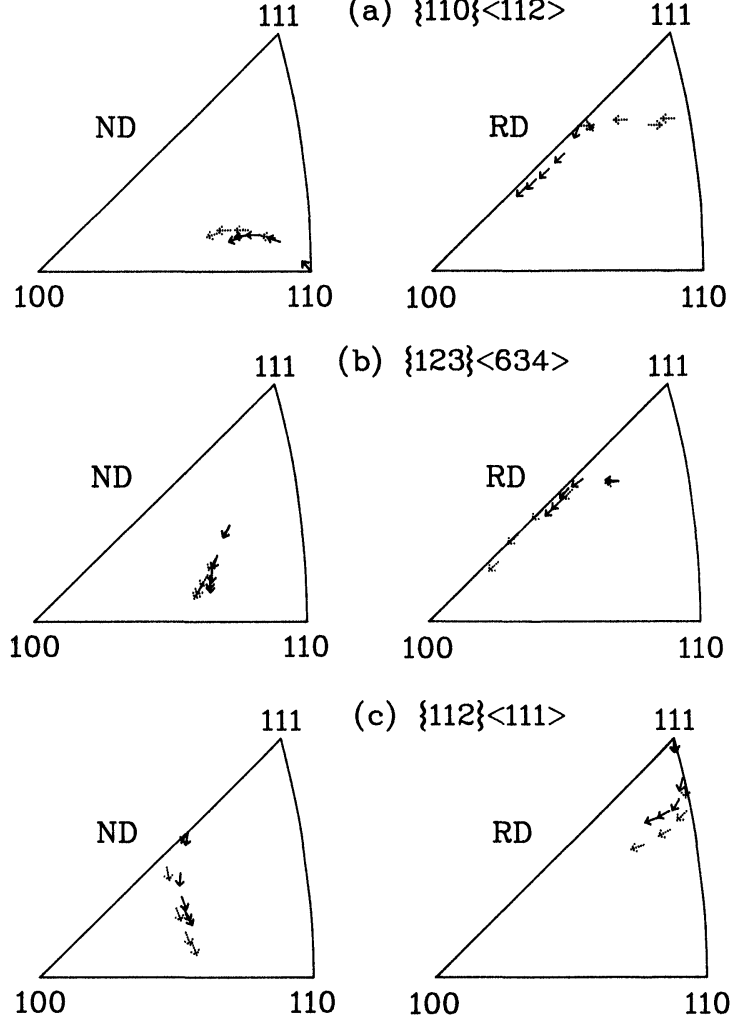

(c) $\{112\}<111>$
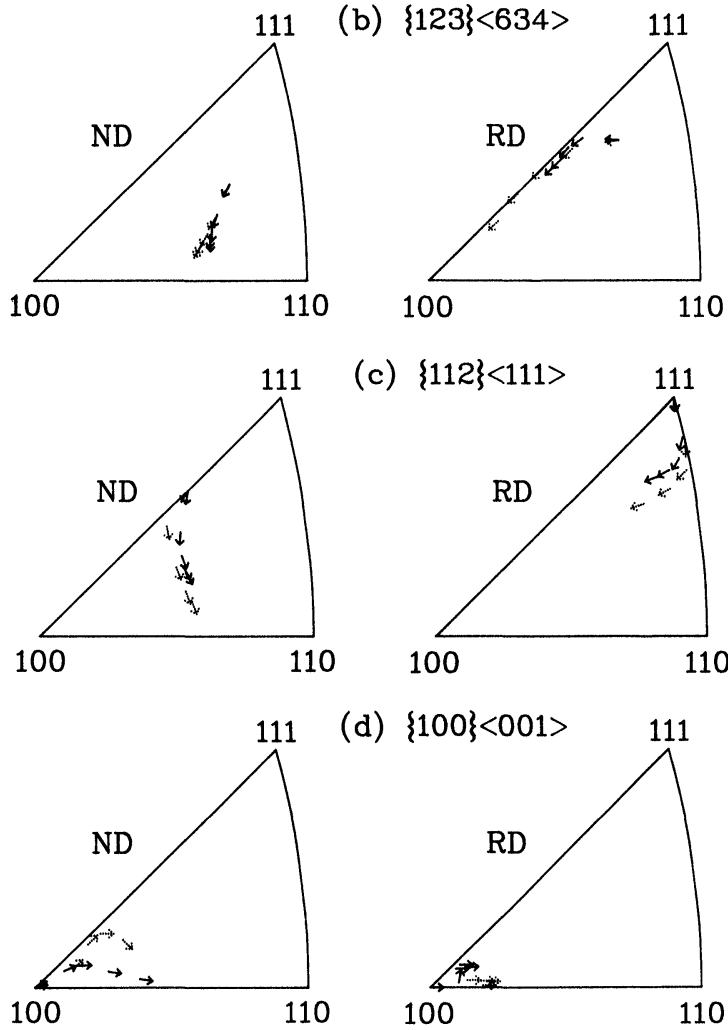

(d) $\{100\}<001>$
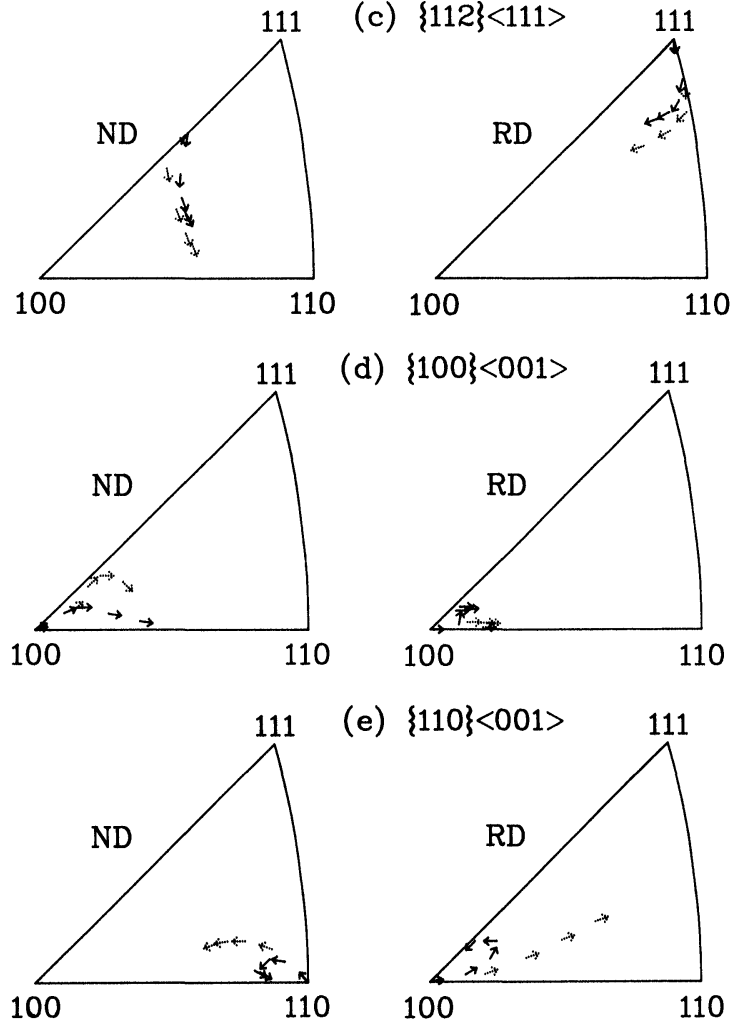

(e) $\{110\}<001>$

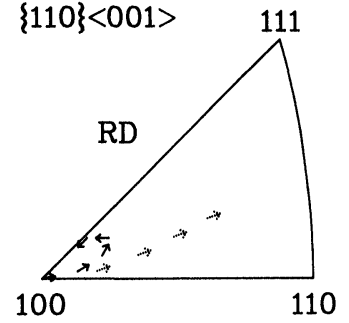

Figure 2 Inverse pole figures for the simulated rotation paths of the texture components of the C.P. aluminium sheet (a) $\{110\}\langle 112\rangle$ (b) $\{123\}\langle 634\rangle$ (c) $\{112\}\langle 111\rangle$ (d) $\{100\}\langle 001\rangle$ (e) $\{110\}\langle 001\rangle$ under non-prescribed strain path $(\rightarrow)$ and prescribed strain path $(\rightarrow)$ under equibiaxial tension. 
(a)

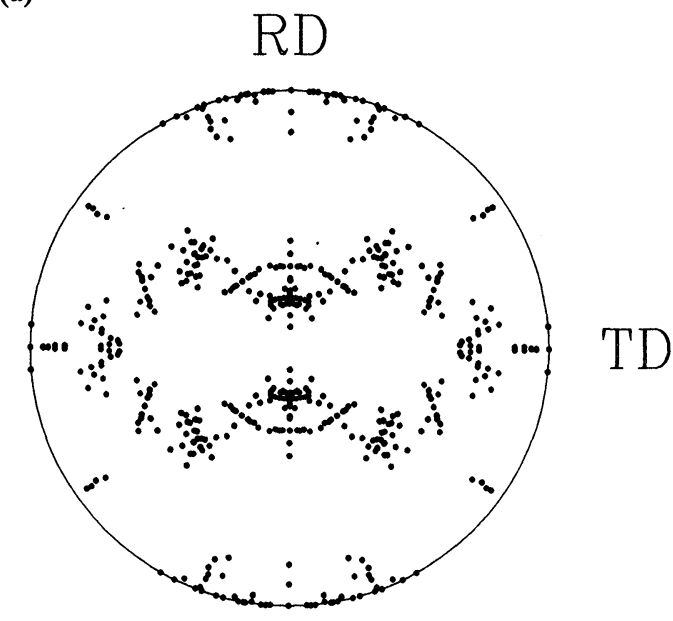

(b)
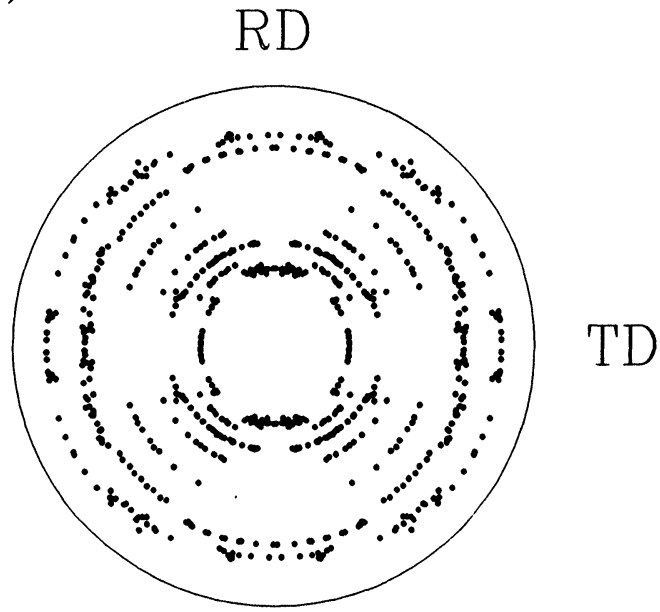

Figure $3\{111\}$ pole figures of (a) simulated starting C.P. aluminium texture, (b) simulated equibiaxial deformation texture at the thickness strain of 0.8 under prescribed strain path.

results obtained by Kohara (1981) in the hydraulic bulging of C.P. aluminium sheet. Despite the general similarities of the two simulated textures, differences exist in the degree of clustering of the orientations which are not revealed by the two dimensional pole-figure. A misorientation analysis was carried out. The misorientation is described exactly by a rotation axis and rotation angle that transforms one orientation into the other. The texture obtained under the non-prescribed strain path is sharper and has more orientations close to $\{110\}\langle 001\rangle$ and $\{110\}\langle 112\rangle$. 52.4\% of grains deformed under the non-prescribed strain paths are within $20^{\circ}$ of the angular spread of $\{110\}\langle 112\rangle$ and $27.1 \%$ of 
(c)

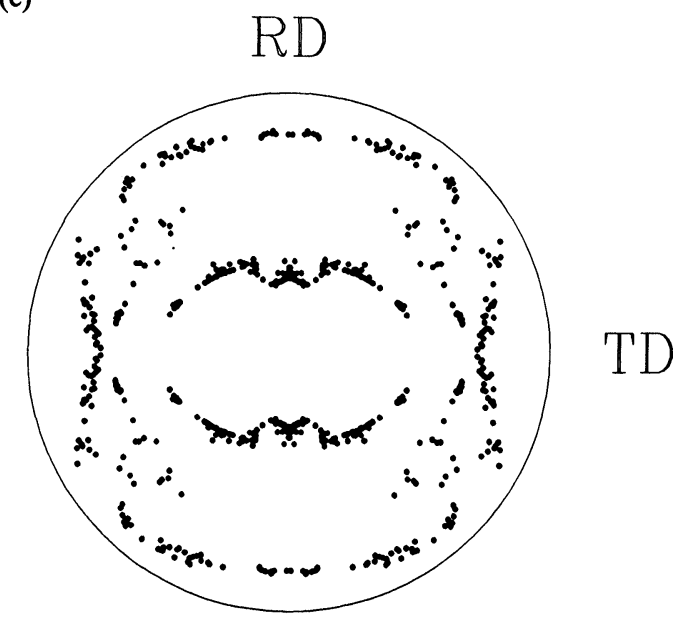

Figure 3 (Continued) $\{111\}$ pole figure of (c) simulated equibiaxial deformation texture at the thickness strain of 0.8 under non-prescribed strain path.

grains are within $20^{\circ}$ of $\{110\}\langle 001\rangle$. The corresponding figures for the grains deformed under a fully prescribed linear strain path are $6.5 \%$ and $18.1 \%$ only.

The above analysis shows that the strain ratio $\left(\epsilon_{11} / \epsilon_{22}\right)$ taken by an anisotropic sheet metal differs from 1 and varies in magnitude in equibiaxial stretching. Dillamore et al. (1971) in their experimental study of the mechanical anisotropy of cubic metals reported a constant strain ratio for constant loading paths. However the strain they used in their experiment was very small $(<0.05)$. This does not contradict the predictions made here where the plastic strain involved is large. The change from a linear strain path to a curvilinear one as a result of the rapid development of the deformation texture in biaxial stretching should not be overlooked. Differences in the rotation path and deformation texture are predicted for sheet metal deformed according to the prescribed or non-prescribed strain path. The texture predicted based on the non-prescribed strain path agrees qualitatively better with the published experimental resuts than the one predicted from the prescribed or fixed strain path.

It would be of interest to compare the prediction of the equibiaxial stretching texture of C.P. aluminium made by Welch (1982) based on the method described by Kallend and Davies (1972). The starting texture was a typical balanced texture of cube components and the pure metal type retained rolling texture components. After a biaxial strain of 0.113 , the experimental results showed that the cube texture was depleted. The maximum in the skeletal line of the orientation tube had moved near the $\{110\}\langle 112\rangle$ orientation. Orientations near $\{110\}\langle 010\rangle$ increased. However, their predicted texture shows an amount of the cube starting-texture and a $\{110\}\langle\mathrm{uvw}\rangle$ fibre texture with maximum at the $\{110\}\langle 110\rangle$ positions. This discrepancy was attributed by Welch to the formation of "looper lines" which are lines of differential thinning occurring parallel to the rolling direction as a result of the non-compliance of individual crystals with the macroscopically imposed shape change. The differences in the prediction between 
this work and Welch's may be due to the different approach in the selection of slip systems. In Welch's work, a random choice was made when more than 5 independent slip systems are available. In this paper the criterion of the minimum second order plastic work of Renouard and Winterberger is used.

\section{CONCLUSION}

The texture evolution of commercially pure aluminium sheet under equibiaxial tension has been analyzed. The following observations are made:

(1) The strain path is found to vary along a constant loading path.

(2) The stability of grain orientation is related to the strain path undertaken.

(3) Differences in equibiaxial deformation textures are predicted between prescribed strain paths and non-prescribed strain paths.

(4) The equibiaxial deformation textures predicted based on non-prescribed strain path agrees better with the experimental results than the one predicted from the prescribed or fixed strain path.

\section{References}

Bacroix, B., Jonas, J. J., Montheillet, F. and Skalli, A. (1986). Acta Metall. 34, 937-950.

Bishop, J. F. W. and Hill, R. (1951a). Phil. Mag. 42, 414-427.

Bishop, J. F. W. and Hill, R. (1951b). Phil. Mag. 42, 1298-1307.

Bunge, H. J. (1969). Texture Analysis in Material Science, Akademie, Berlin.

Bunge, H. J. (1971). Kristall u. Technik. 5, 145-175.

Chan, K. C. and Lee, W. B. (1990). A paper accepted for publication in Int. J. Mech. Sci. 32.

Dillamore, I. L., Hazel, R. J., Watson, T. W. and Hadden, P. (1971). Int. J. Mech. Sci. 13, 1049-1061.

Driver, J. H., Skalli, A. and Wintenberger, M. (1983). Mém. scient, Revue Métall. 80, 241.

Hansen, N. and Jensen, D. J. (1986). Met. Trans. A 17A, 253-259.

Honneff, H. and Mecking, H. (1978). Textures of Materials (edited by G. Gottstein and K. Lücke), Springer, Berlin, pp. 265-275.

Kallend, J. S. and Davies, G. J. (1972). Phil. Mag. 25, 471-490.

Kocks, U. F. (1975). Constitutive Relations for Slip, in Constitutive Equations in Plasticity (edited by A. S. Argon), MIT Press, Mass, pp. 81-116.

Kohara, S. (1981). Proc. 6th Int. Conf. in Texture of Mat., Tokyo, vol. 1, pp. 300-307.

Lequeu, Ph., Montheillet, F. and Jonas, J. J. (1986). Symp. on computer Modelling of Fabrication Processes and Constitutive Behaviour of Metals, Canada, pp. 237-265.

Montheillet, F., Gilormini, P. and Jonas, J. J. (1985). Acta Metall. 33, 705-717.

Ragab, A. R. and Abbas, A. T. (1986). J. Eng. Mat'l and Tech. 108, 251-266.

Renouard, M. and Wintenberger, M. (1981). C.r. Acad. Sci. Paris, B292, 385-388.

Sachs, G. (1928). Z. d. Ver. deut. Ing. 72, 734-736.

Taylor, G. I. (1938). J. Inst. Met. 62, 307-324.

Van Houtte, P. and Aernoudt, E. (1975). Z. Metallk. 66, 202-209.

Vial, C. and Hosford, W. F. (1983). Int. J. Mech. Sci. 25, 899-915.

Welch, P. I. (1982). Z. Metallk. 73, 381-386.

\section{APPENDIX}

Notations used in the formulae

б stress tensor

€ strain tensor 


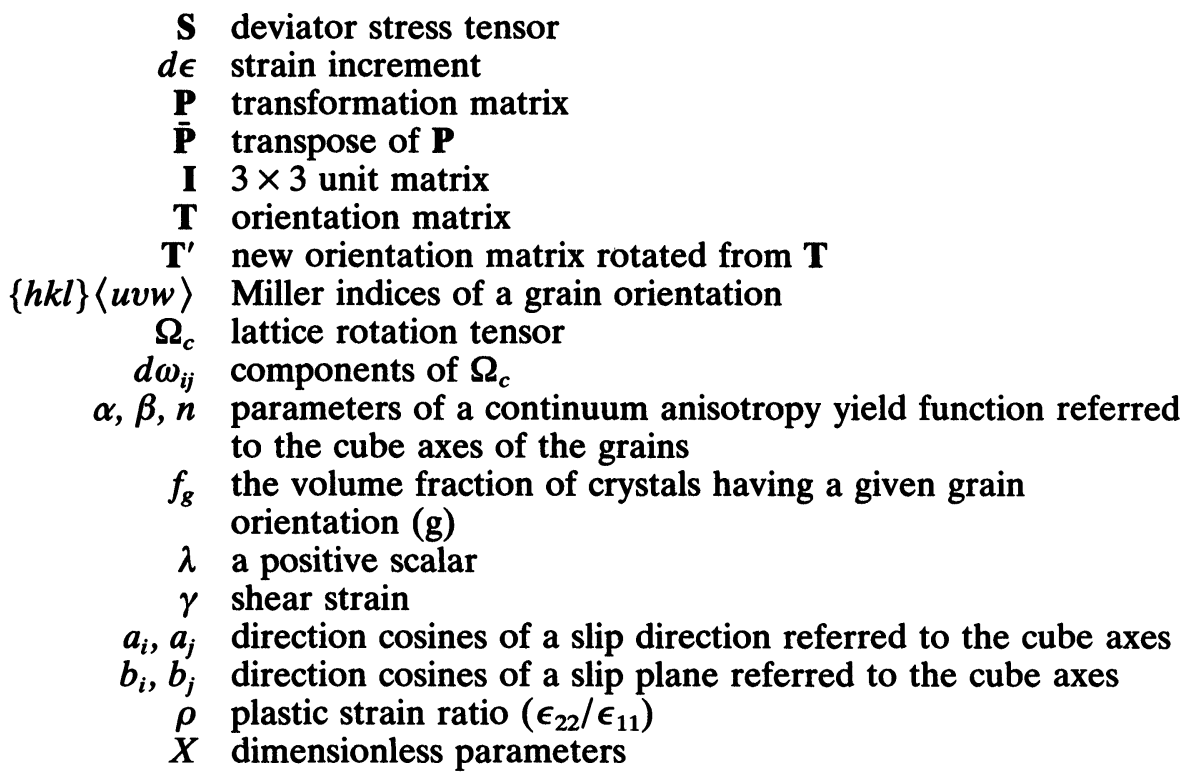

Subscripts

ij components of a tensor or a matrix

$c$ referred to the $\langle 100\rangle$ crystal axes

Superscripts

$g$ referred to an ideal grain orientation 